\title{
THE MORSE INDEX THEOREM IN THE DEGENERATE ENDMANIFOLD CASE
}

\author{
NANCY HINGSTON AND DIANE KALISH
}

(Communicated by Peter Li)

\begin{abstract}
We present a simple formulation and proof of the Morse index theorem for two endmanifolds in the degenerate case, that is, when each submanifold lies at a focal point of the other.
\end{abstract}

\section{INTRODUCTION}

Let $M$ be a Riemannian manifold with submanifolds $P$ and $Q$. The energy function $E$ is defined on the space $\Omega(M ; P, Q)$ of continuous piecewise $C^{\infty}$ paths joining $P$ and $Q$. A path $\gamma \in \Omega(M ; P, Q), \gamma:[0, T] \rightarrow M$, is a critical point for $E$ when $\gamma$ is a geodesic intersecting $P$ and $Q$ orthogonally at $\gamma(0)$ and $\gamma(T)$. Let $H$ be the linear space of continuous, piecewise $C^{\infty}$ vector fields along and orthogonal to $\gamma$ whose initial and final vectors are in $P_{\gamma(0)}$ and $Q_{\gamma(T)}$. The Morse index form is a symmetric bilinear map $I: H \times H \rightarrow \mathbb{R}$.

In [4] the index of $I$ at a critical point $\gamma$ was described, provided the endpoint $\gamma(T)$ was not a $P$-focal point. The index was computed using $P$-focal points and a calculation at $\gamma(T)$ involving the second fundamental form of $Q$. This paper extends the proof to include the case when $\gamma(T)$ is a $P$-focal point. To accomplish this, we add a third term in the index calculation, which we call the $Q$-multiplicity of the $P$-focal point $\gamma(T)$.

Definitions. Many of the basic definitions can be found in [4]. Let $M, P, Q, \gamma$, and $H$ be as in the introduction. The Morse index form $I$ is given by

$$
\begin{aligned}
I(X, Y)= & \int_{0}^{T}\left\langle R X(t)-X^{\prime \prime}(t), Y(t)\right\rangle d t+\sum_{i}\left\langle X^{\prime}\left(t_{i}^{-}\right)-X^{\prime}\left(t_{i}^{+}\right), Y\left(t_{i}\right)\right\rangle \\
& +\left.\left\langle X^{\prime}(t)-S_{t} X(t), Y(t)\right\rangle\right|_{0} ^{T},
\end{aligned}
$$

where the $t_{i}$ are the points of discontinuity of $X^{\prime}$ in $(0, T) ; S_{0}$ is the second fundamental form of $P$ at $\gamma(0)$ with respect to $\gamma^{\prime}(0)$, and $S_{T}$ is the second fundamental form of $Q$ at $\gamma(T)$ with respect to $\gamma^{\prime}(T)$.

A $P$-Jacobi field is a Jacobi field $J$ that is orthogonal to $\gamma$ with $J(0) \in P_{\gamma(0)}$ and with $J^{\prime}(0)-S_{0} J(0) \perp P_{\gamma(0)}$; thus $J$ corresponds to a variation by geodesics

Received by the editors October 11, 1991.

1991 Mathematics Subject Classification. Primary 58E05.

The second author's research was partially supported by the School of Science and Mathematics of William Paterson College under the Governor's Challenge Grant. 
leaving $P$ orthogonally. A symmetric bilinear map $A$ is defined on the space of $P$-Jacobi fields whose value at $T$ is contained in $Q_{\gamma(T)}$ by

$$
A(X, Y)=\left\langle X^{\prime}(T)-S_{T} X(T), Y(T)\right\rangle .
$$

Let $M_{T}$ denote $\left(\gamma^{\prime}(T)\right)^{\perp}$. W will be the subspace of $M_{T}$ spanned by the values of the $P$-Jacobi fields at $\gamma(T)$, and $W^{\perp}$ will be the orthogonal complement of $W$ in $M_{T}$. Then $W^{\perp}$ is spanned by the values at $T$ of the derivatives of the $P$-Jacobi fields vanishing at $T$.

The $Q$-multiplicity $m_{T}$ of $\gamma(T)$ as a $P$-focal point is defined to be the dimension of the orthogonal projection $\operatorname{Proj}_{Q}$ of $W^{\perp}$ onto $Q_{\gamma(T)}$. Alternatively,

$$
m_{T}=\operatorname{dim} Q-\operatorname{dim}\left(W \cap Q_{\gamma(T)}\right) .
$$

Thus, for example, if $Q$ is a point then $m_{T}=0$; if $Q$ is a hypersurface, then $m_{T}$ is the multiplicity of $\gamma(T)$ as a $P$-focal point in the ordinary sense, i.e., $\operatorname{dim} W^{\perp}$.

Statement of the index theorem. The index of $I$ is given by the sum of the index of $A$, the $Q$-multiplicity of $\gamma(T)$ as a P-focal point, and the sum of the multiplicities of the P-focal points before $\gamma(T)$. That is,

$$
\operatorname{index}(I)=\operatorname{index}(A)+m_{T}+\sum m_{i} \text {. }
$$

Here $m_{i}$ is the multiplicity of the P-focal point $\gamma\left(t_{i}\right), 0<t_{1}<\cdots<t_{k}<T$.

The formula for the augmented index is simpler:

$$
(\text { index }+ \text { nullity })(I)=(\text { index }+ \text { nullity })(A)+\sum m_{i} .
$$

Remarks. The computation of the augmented index follows easily from that of the index. First, it is well known [2] that the nullspace of $I$ is the space of simultaneous $P$-and $Q$-Jacobi fields, i.e., the space of $P$-Jacobi fields $X$ with $X(T) \in Q_{\gamma(T)}$ and $X^{\prime}(T)-S_{T} X(T) \perp Q_{\gamma(T)}$. The nullspace of $A$ is the space of $P$-Jacobi fields $X$ with $X(T) \in Q_{\gamma(T)}$ and $X^{\prime}(T)-S_{T} X(T) \perp W \cap Q_{\gamma(T)}$. Consider the linear map on the nullspace of $A$ taking $X$ to the orthogonal projection of $X^{\prime}(T)-S_{T} X(T)$ onto $Q_{\gamma(T)}$. The kernel of this map is the nullspace of $I$. It is not hard to see that the image of this map is precisely $\operatorname{Proj}_{Q} W^{\perp}$. Thus

$$
\operatorname{Nullity}(A)=m_{T}+\operatorname{Nullity}(I) .
$$

Readers familiar with the case considered by Morse, i.e., when $Q$ is a point, will wonder how the index varies as we "move" $Q$ along $\gamma(t), 0<t \leq T$. Other authors $[1,3]$ have studied the problem from this point of view, by (in different ways) translating the boundary condition represented by $Q$ along $\gamma$. This leads to a more complicated statement and a more complicated proof. We prove the theorem without any such translation; however, we will now give a rough explanation of how the index varies as we move $Q$ in the case when $Q$ is a hypersurface. The "orthogonal" case when $Q$ is a point is well known. The general case lies somewhere in between; while no essentially new phenomena occur, the general case is technically complicated for reasons mentioned below. The following discussion should also make clear how our statement is equivalent to that of Bolton [3] in the hypersurface case. Note that Bolton's theorem is more restrictive than ours as his formulation excludes degenerate $P$ - $Q$ focal points. 
Let $Q$ be a hypersurface in $M$ and imagine $Q$ being translated along $\gamma$ in some way so that the tangent space $Q_{t}$ at $\gamma(t)$ is orthogonal to $\gamma^{\prime}(t)$. Let $A_{t}, W_{t}, M_{t}, I_{t}$, and $m_{t}$ correspond to $A, W, M_{T}, I$, and $m_{T}$. Then $m_{t}=$ $\operatorname{dim} W_{t}^{\perp}$ is the multiplicity of $\gamma(t)$ as a $P$-focal point, and $A_{t}$ is defined for each $t$ on the entire space of $P$-Jacobi fields. On an interval with no $P$-focal points, the index of $I_{t}$ is locally constant except where the index of $A_{t}$ changes. This will happen only at points where $A_{t}$ has nonzero nullity. The nullity of $A_{t}$ at a non- $P$-focal point $\gamma(t)$ is the dimension of the space of simultaneous $P$ and $Q(t)$-Jacobi fields (corresponding to variations of $\gamma$ by geodesics leaving $P$ and arriving at $Q(t)$ orthogonally). Bolton calls such points $\gamma(t)$ signed $(P, Q)$ focal points; the sign tells whether the index of $A_{t}$ (and thus of $I_{t}$ ) increases or decreases.

When $Q$ is a hypersurface, the nullspace of $A_{t}$ at a $P$-focal point has a subspace of dimension $m_{t}$ coming from focal $P$-Jacobi fields. Thus $\operatorname{Nullity}\left(A_{t}\right) \geq$ $m_{t}$; in fact, the index of $I_{t}$ will be constant as we pass through a focal point as long as $\operatorname{Nullity}\left(A_{t}\right)=m_{t}$, i.e., unless two things are happening at once: Let $\gamma(s)$ be a focal point with $\operatorname{Nullity}\left(A_{s}\right)=m_{s}$. We claim that

$$
\operatorname{index}\left(A_{s+\varepsilon}\right)=\operatorname{index}\left(A_{s-\varepsilon}\right)-m_{s} .
$$

Thus in our computation index $\left(A_{t}\right)$ decreases by $m_{s}$ and $\sum m_{i}$ increases by $m_{s}$, keeping index $\left(I_{t}\right)$ constant. This is not hard to see using the following: Let $X$ be a $P$-Jacobi field with $X(s)=0$. Assuming $S_{t}$ is continuous, we have

$$
A_{t}(X, X)=\left\langle X^{\prime}(s), X^{\prime}(s)\right\rangle(t-s)+\mathrm{rem}
$$

with a standard Taylor-type remainder.

Here is a simple example: Let $M=\mathbb{R}^{2}, P=\left\{x^{2}+y^{2}=4 ; x \leq 0\right\}$, and let $Q(t)=\left\{(x-t)^{2}+y^{2}=1, x-t \leq 0\right\}, \gamma_{t}=\{(r, 0) \mid-2 \leq r \leq t-1\}$ (see Figure $1)$.

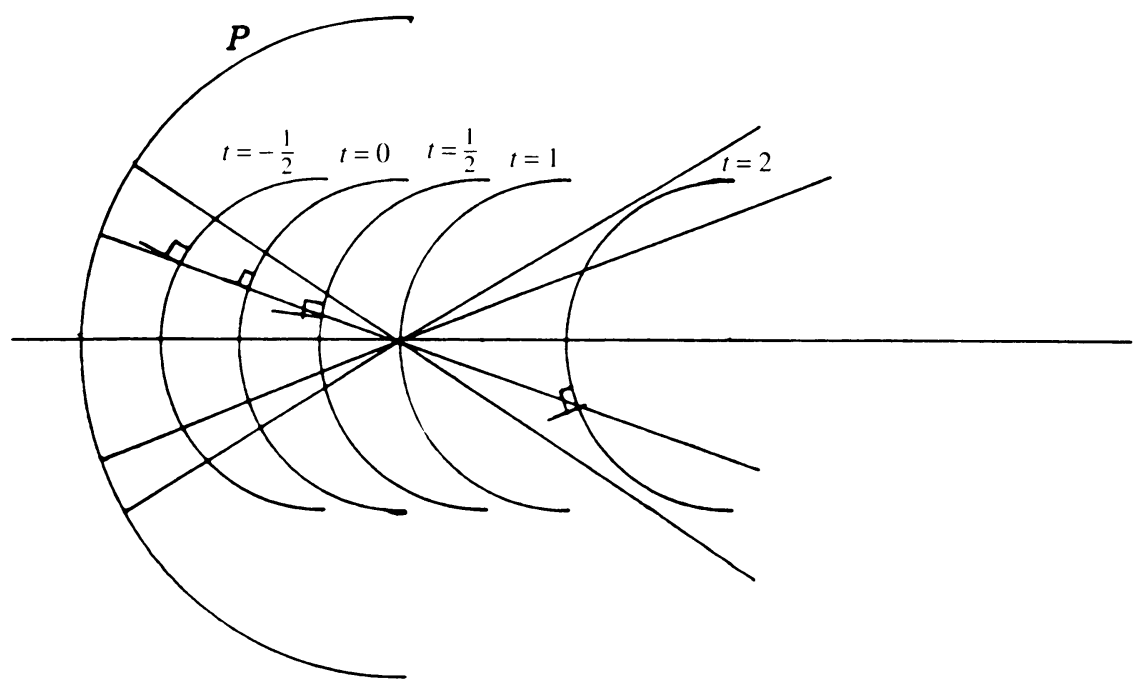

FIGURE 1 
Then

$$
\begin{array}{lllll}
\text { for }-1<t<0, & A_{t}>0, & m_{t}=0, & \sum m_{i}=0, & I_{t}=0 ; \\
\text { for } t=0, & A_{t}=0, & m_{t}=0, & \sum m_{i}=0, & I_{t}=0 ; \\
\text { for } 0<t<1, & A_{t}<0, & m_{t}=0, & \sum m_{i}=0, & I_{t}=1 ; \\
\text { for } t=1, & A_{t}=0, & m_{t}=1, & \sum m_{i}=0, & I_{t}=1 ; \\
\text { for } t>1, & A_{t}>0, & m_{t}=0, & \sum m_{i}=1, & I_{t}=1 .
\end{array}
$$

Here $t=0$ gives a simultaneous $P$ - and $Q(T)$-Jacobi field and is the point where the index changes; $t=1$ is the focal point. The sign of $A_{t}$ depends upon the angle made by the geodesics leaving $P$ orthogonally with the normal vector to $Q(t)$, since this angle determines whether these geodesics will be longer or shorter than $\gamma_{t}$.

Note: in general we have an orthogonal decomposition

$$
M_{t}=\underbrace{W_{t} \cap Q_{t} \oplus \operatorname{Proj}_{Q_{t}}\left(W_{t}^{\perp}\right)}_{Q_{t}} \oplus \underbrace{Q_{t}^{\perp} \cap W_{t}^{\perp} \oplus \operatorname{Proj}_{Q_{t}^{\perp}}\left(W_{t}\right)}_{Q_{t}^{\perp}} .
$$

The dimension of the middle two terms is the multiplicity of $\gamma(t)$ as a $P$-focal point and the dimension of the second term is $m_{t}$, the $Q(t)$-multiplicity of $\gamma(t)$ as a $P$-focal point. The domain of $A_{t}$ is a vector subspace of the space of $P$-Jacobi fields whose dimension is that of the first three terms. When $Q$ is a hypersurface, the last two terms vanish; when $Q$ is a point, the first two terms vanish. Roughly speaking, we see "hyperspace" phenomena in the first two terms and "point" phenomena in the last two terms. However, this becomes technically messy to write down because the domain of $A_{t}$ is not a constant subspace even away from focal points.

\section{PROOF OF THE THEOREM}

As in [4], our aim will be to write $H=B \oplus B^{c}$, where $B$ is the subspace of $H$ consisting of vector fields in $H$ whose values at the $P$-focal points in $(0, T)$ are in the span of the values of the $P$-Jacobi fields at these points and whose value at $T$ is zero. By Lemma 2 in [4], $I$ is nonnegative on $B$. As in [4], we will find the index of $I$ on $B^{c}$, but our definition of $B^{c}$ will have a slightly different formulation.

Since

$$
M_{T}=Q_{\gamma(T)} \oplus Q_{\gamma(T)}^{\perp}=W \oplus W^{\perp},
$$

it follows that

$$
Q_{\gamma(T)}=W \cap Q_{\gamma(T)} \oplus \operatorname{Proj}_{Q}\left(W^{\perp}\right) .
$$

Let $s$ be the dimension of $W \cap Q_{\gamma(T)}$ and $r$ the dimension of $Q_{\gamma(T)}$. Let $K_{1}, \ldots, K_{s}$ be $P$-Jacobi fields whose values at $\gamma(T)$ span $W \cap Q_{\gamma(T)}$, and let $Y_{T}^{1}, \ldots, Y_{T}^{r-s}$ be $P$-Jacobi fields that vanish at $\gamma(T)$ and such that the orthogonal projections of their derivatives at $T$ onto $Q_{\gamma(T)}$, together with $K_{1}(T), \ldots, K_{s}(T)$, span $Q_{\gamma(T)}$. We can assume that these projections are orthonormal. We recall from [4] the definition of the $V_{i}^{j}$ : at the $P$-focal point 

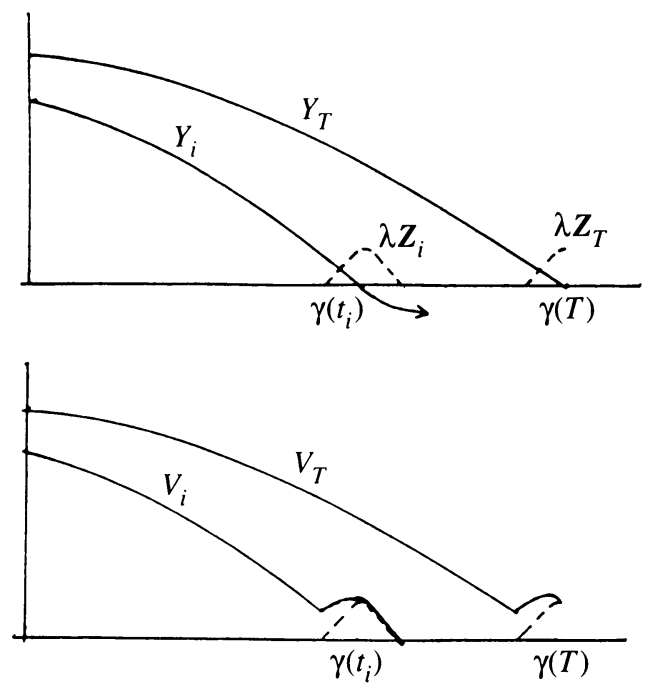

FIGURE 2

$\gamma\left(t_{i}\right), 0<t_{i}<T$, the $Y_{i}^{j}, 1 \leq j \leq m_{i}$, are $P$-Jacobi fields that vanish at $t_{i}$ with $\left\langle\left(Y_{i}^{j}\right)^{\prime}\left(t_{i}\right),\left(Y_{i}^{k}\right)^{\prime}\left(t_{i}\right)\right\rangle=\delta_{j k} . \hat{Y}_{i}^{j}=Y_{i}^{j}$ on $\left[0, t_{i}\right]$ and $\hat{Y}_{i}^{j}=0$ elsewhere. The $Z_{i}^{j}$ are bump functions with small support and value $-\left(Y_{i}^{j}\right)^{\prime}\left(t_{i}\right)$ at $t_{i}$ (see Figure 2). For $\lambda>0$ put

$$
V_{i}^{j}=\widehat{Y}_{i}^{j}+\lambda Z_{i}^{j}, \quad 1 \leq i \leq k, 1 \leq j \leq m_{i} .
$$

Here $k$ is the number of $P$-focal points before $\gamma(T)$.

Let $V_{T}^{1}, \ldots, V_{T}^{r-s}$ be constructed in the same way as $V_{i}^{j}$, except let $Z_{T}^{j}(T)$ be the orthogonal projection onto $Q_{\gamma(T)}$ of the $-\left(Y_{T}^{j}\right)^{\prime}(T), 1 \leq j \leq r-s$.

Definition of $B^{c}$. Let $B^{c}$ denote the span of the $V_{i}^{j}$, the $V_{T}^{j}$, and the $K_{l}$. As in [4], we have the

Claim. (1) The dimension of $B^{c}$ is $r+\sum_{i=1}^{k} m_{i}$.

(2) $H=B \oplus B^{c}$.

(3) $I(X, X) \geq 0$ for $X \in B$.

Let $N$ be the index of $A$. We can choose the $K_{l}$ so that $A$ is negative definite $(<0)$ on $K_{1}, \ldots, K_{N}$ and positive $(\geq 0)$ on $K_{N+1}, \ldots, K_{s}$.

Let $B_{-}^{c}=\operatorname{Span}\left\{K_{1}, \ldots, K_{N}, V_{i}^{j}, V_{T}^{j}\right\}$ and $B_{+}^{c}=\operatorname{Span}\left\{K_{N+1}, \ldots, K_{s}\right\}$. Then $B^{c}=B_{-}^{c} \oplus B_{+}^{c}$ and $H=B \oplus B_{+}^{c} \oplus B_{-}^{c}$. As before, $I\left(B_{+}^{c}, B_{+}^{c}\right) \geq 0$ and $I\left(B, B_{+}^{c}\right)=0$; thus $I$ is positive $(\geq 0)$ on $B \oplus B_{+}^{c}$. Thus to prove the theorem it remains to prove the

Lemma. If $\lambda$ is sufficiently small, $I(X, X)<0$ for $X \in B_{-}^{c}$.

Proof. Take a metric on $B_{-}^{c}$ for which the given spanning vectors are orthonormal. With respect to this basis, $I$ is represented by a matrix of the form

$$
\left(\begin{array}{cc}
N & \lambda D \\
\lambda D & -2 \lambda U+\lambda^{2} C
\end{array}\right),
$$

where $U$ is the matrix representing the identity map on $\operatorname{Span}\left\{V_{i}^{j}, V_{T}^{j}\right\}$ and $N$ (representing $A$ on $\left.\operatorname{Span}\left\{K_{1}, \ldots, K_{N}\right\}\right)$ is negative definite. Here $C$ is the 
matrix whose entries are the $I\left(Z_{i}^{j}, Z_{k}^{l}\right) ; D, C$, and $N$ are independent of $\lambda$. The computation is similar to that in [4] except that $D$ is no longer 0 ; it has nonzero entries given by

$$
I\left(K_{l}, V_{T}^{j}\right)=\lambda\left\langle K_{l}^{\prime}(T)-S_{T} K_{l}(T),-\operatorname{Proj}_{Q}\left(Y_{T}^{j}\right)^{\prime}(T)\right\rangle .
$$

To show that $I$ is negative definite on $B_{-}^{c}$, it is sufficient to show that it is negative definite on every two-dimensional subspace spanned by unit vectors $k \in \operatorname{Span}\left\{K_{1}, \ldots, K_{N}\right\}$ and $v \in \operatorname{Span}\left\{V_{i}^{j}, V_{T}^{j}\right\}$. The matrix of the restriction of $I$ to this subspace has a matrix (using $k$ and $v$ as basis)

$$
\left(\begin{array}{cc}
-n^{2} & \lambda d \\
\lambda d & -2 \lambda+\lambda^{2} c
\end{array}\right)
$$

that is negative definite (determinant $>0$ and trace $<0$ ) for $\lambda$ sufficiently small. As $k$ and $v$ range over the unit spheres in $\operatorname{Span}\left\{K_{1}, \ldots, K_{N}\right\}$ and $\operatorname{Span}\left\{V_{i}^{j}, V_{T}^{j}\right\}$, we can find a $\lambda>0$ so that this will always be true.

Thus the theorem is proved.

\section{REFERENCES}

1. W. Ambrose, The index theorem in Riemannian geometry, Ann. of Math. (2) 73 (1961), 49-86.

2. R. L. Bishop and R. L. Crittenden, Geometry of manifolds, Academic Press, New York, 1964.

3. J. Bolton, The Morse Index Theorem in the case of two variable end-points, J. Differential Geom. 12 (1977), 567-581.

4. D. Kalish, The Morse index theorem where the ends are submanifolds, Trans. Amer. Math. Soc. 308 (1988), 341-348.

Department of Mathematics, Trenton State College, Trenton, New Jersey 08650

Department of Mathematics, William Paterson College, Wayne, New Jersey 07470 